\title{
What Are Risk Factors for Intraoperative Humerus Fractures During Revision Reverse Shoulder Arthroplasty and Do They Influence Outcomes?
}

\author{
Eric R. Wagner MD, Matthew T. Houdek MD, Bassem T. Elhassan MD, \\ Joaquin Sanchez-Sotelo MD, PhD, Robert H. Cofield MD, \\ John W. Sperling MD, MBA
}

Received: 17 December 2014/ Accepted: 30 June 2015/Published online: 11 July 2015

(C) The Association of Bone and Joint Surgeons $\mathbb{R} 2015$

\begin{abstract}
Background With the increase in shoulder arthroplasty rates, the number of perioperative complications, such as periprosthetic fractures, continues to be a rise; however, the risk factors and incidence of intraoperative complications, such as fractures, during revision reverse shoulder arthroplasty are not well established.

Questions/purposes We evaluated patients receiving a reverse shoulder arthroplasty to determine (1) the frequency and characteristics of intraoperative humerus fractures, (2) the risk factors for fracture, (3) the complications associated with treatment of fractures, and (4) clinical and functional outcomes after treatment.

Methods Using one institution's total joint registry, we performed a retrospective analysis of medical records of 224 patients (230 shoulders) who underwent revision surgery to reverse shoulder arthroplasty, from 2005 to 2012, for failed
\end{abstract}

One of the authors certifies that he (JWS) has received or may receive payments or benefits, during the study period, an amount of USD 10,000 to USD 100,000 from Biomet, Inc (Warsaw, IN, USA). All ICMJE Conflict of Interest Forms for authors and Clinical Orthopaedics and Related Research ${ }^{\circledR}$ editors and board members are on file with the publication and can be viewed on request.

Clinical Orthopaedics and Related Research ${ }^{\mathbb{R}}$ neither advocates nor endorses the use of any treatment, drug, or device. Readers are encouraged to always seek additional information, including FDA approval status, of any drug or device before clinical use.

Each author certifies that his or her institution approved the human protocol for this investigation and that all investigations were conducted in conformity with ethical principles of research.

E. R. Wagner, M. T. Houdek, B. T. Elhassan, J. Sanchez-Sotelo, R. H. Cofield, J. W. Sperling ( $₫)$

Department of Orthopedic Surgery, Mayo Clinic, 200 First Street

SW, Rochester, MN 55905, USA

e-mail: Sperling.john@mayo.edu total shoulder arthroplasty. Reverse shoulder arthroplasty was used when there was a deficient rotator cuff, prior instability, or deficient glenoid bone stock. Intraoperative periprosthetic humerus fractures occurred in 36 shoulders (16\%) (36 patients). The clinical outcome analysis included 29 patients with a minimum 2-year clinical followup (mean followup, 3.1 years; range, 2.0-6.3 years). The control group consisted of 188 patients (194 shoulders), and it was used for risk-factor calculation; whereas only 150 patients (154 shoulders) in the control group had a minimum 2-year followup, and thus only 150 patients (154 shoulders) made up the comparators (controls) for outcome-analysis comparisons. Risk factors were assessed using univariate analysis with odds ratios (OR), whereas implant survival and complications were assessed using the Kaplan-Meier method.

Results Three displaced and 33 nondisplaced fractures occurred during revision reverse total shoulder arthroplasties. Most of the fractures $(81 \%)$ occurred during component removal of cemented $(\mathrm{n}=11)$ and cementless $(n=25)$ components. Intraoperative fractures only were treated with stabilization of the prosthetic stem in 28 patients, while adjunctive internal fixation was used in eight patients. Risk of intraoperative periprosthetic fractures was increased by factors including female sex ( $\mathrm{n}=18$ women; OR, 2.41; range, 1.11-5.68; $\mathrm{p}=0.03$ ); history of instability $(\mathrm{n}=27$; OR, 2.65; range, $1.18-5.93 ; \mathrm{p}=0.02)$; and prior hemiarthroplasty $(\mathrm{n}=22$; OR, 2.34; range, $1.13-4.84 ; \mathrm{p}=0.03$ ). There were two postoperative fractures in patients who had an intraoperative fracture and both were treated nonoperatively. Overall, three $(8 \%)$ revision procedures were performed in patients with intraoperative fractures, with 2- and 5-year survivorship estimates of $94 \%$ and $85 \%$, respectively, compared with $89 \%$ and $84 \%$, respectively for patients without an intraoperative fracture $(\mathrm{p}=0.45)$. At latest followup, patients 
experienced good postoperative pain relief, improved shoulder abduction, and good American Shoulder and Elbow Surgeon and Simple Shoulder Test scores.

Conclusions Intraoperative humeral fractures occur in approximately $16 \%$ of shoulders undergoing revision surgery. Fractures during revision reverse TSA are not uncommon secondary to the risks of component removal in revision surgery and poor remaining bone stock. The risk seems to be greatest for female patients, patients with instability, and patients who have undergone previous hemiarthroplasties. Intraoperative humeral fractures should be approached in a systematic way to achieve anatomic reduction and stable fixation. When properly stabilized, these fractures appear not to substantially influence overall final outcome. This study provides a foundation for future investigation of methods to reduce the risk for intraoperative humeral fractures attributable to reverse revision TSA. Level of Evidence Level III, therapeutic study.

\section{Introduction}

There has been a steady increase in the number of total shoulder arthroplasties (TSA) performed in the United States, with relatively low overall complication rates [21]. Periprosthetic fractures associated with anatomic shoulder arthroplasty are relatively uncommon, with an incidence between $0.6 \%$ and $3 \%$ [1, 6, 8, 9, 12, 15, 18, 19, 24-26]. Although not studied as comprehensively as postoperative fractures, the incidence, risk factors, and outcomes associated with intraoperative periprosthetic fractures during anatomic shoulder arthroplasty has been characterized [1, 19]. The rate of intraoperative fractures during revision primary anatomic arthroplasty is as much as 3.3\% [1]. Intraoperative periprosthetic fractures increase operative time, alter implant choices, increase the need for bone grafts, and may have an effect on postoperative outcomes.

The reverse shoulder arthroplasty has improved treatment of patients with glenohumeral arthritis or prior failed shoulder arthroplasty associated with rotator cuff disorders. Although studies have shown good short- to medium-term outcomes for reverse TSAs, there are relatively high incidences of peri- and postoperative complications in the primary setting $[2,11-14,16,20,22]$. Despite these case series, no studies to our knowledge have discussed complications associated with revision reverse TSA. Specifically, little is known regarding the incidence and outcomes associated with intraoperative periprosthetic humerus fractures in reverse TSAs.

The purpose of our investigation was to evaluate patients receiving a reverse TSA to determine (1) the frequency and characteristics of intraoperative humerus fractures, (2) the risk factors for fractures, (3) the complications associated with treatment of fractures, and (4) the clinical and functional outcomes after treatment.

\section{Methods}

After obtaining approval from the institutional review board, we performed a retrospective review using our institution's total joint registry to review the medical records of all patients undergoing a revision surgery to reverse shoulder arthroplasty for a failed previous anatomic or reverse shoulder arthroplasty.

From a medical record review between 2005 and 2012, 224 patients ( 230 shoulders) met our inclusion criterion of having had revision surgery to a reverse shoulder arthroplasty. A reverse shoulder arthroplasty was performed when the patient had a deficient rotator cuff, previous instability, or deficient glenoid bone stock. Any patient with severe humeral bone loss in whom we had performed an allograft prosthetic composite or a proximal humeral replacement prosthesis was excluded from the current study. Five fellowship-trained surgeons (JWS, RHC, JSS, BTE, SPS) were involved in the surgeries. The average age of the 224 eligible patients was 69 years (range, 39-89 years) and average BMI was $31 \mathrm{~kg} / \mathrm{m}^{2}$ (range, $15-48 \mathrm{~kg} / \mathrm{m}^{2}$ ), with women comprising $41 \%$ of the patients. In addition to glenoid loosening and rotator cuff disorders $(\mathrm{n}=73), 27$ patients $(12 \%)$ underwent revision for infection and $57 \%(\mathrm{n}=130)$ had a history of subluxation or instability. Intraoperative periprosthetic humerus fractures occurred in $36(16 \%)$ shoulders (36 patients), while 194 shoulders (188 patients) did not have a fracture. Any patient unwilling to participate in followup through the registry was excluded (while the total number is unavailable, this usually is less than $1 \%$ of our registry patients).

For the clinical analysis, we assessed the group with intraoperative fractures $(n=36$ patients total: 29 patients with more than 2 years followup, seven patients excluded) and compared it with the control group ( $\mathrm{n}=188$ patients total: 150 with more than 2 years followup, 38 excluded). Of the 36 patients with shoulders with intraoperative fractures, the postoperative clinical outcome analysis included only 29 with an intraoperative fracture and who had maintained a minimum 2-year clinical followup. The mean followup was 3.1 years (range, $2-6.3$ years). The control group consisted of 150 patients (154 shoulders) who had not experienced an intraoperative humerus fracture and with a minimum of 2 years clinical followup 
(mean followup, 2.9 years). Thirty-eight of the 188 (40 of the 194 shoulders) patients without fractures were lost to followup and therefore excluded.

\section{Clinical and Radiographic Outcomes}

Demographic information, comorbidities, surgical indications, prior surgical details, and operative findings were collected by a retrospective review of patients' medical records. The findings were used to determine risk stratification for intraoperative periprosthetic fractures. Through the total joint registry, patients completed questionnaires related to those of the American Shoulder and Elbow Surgeons and the Simple Shoulder Test during their followup visit [17]. Results from the patients' last clinic visit combined with the completed questionnaires were used to calculate their pre- and postoperative shoulder pain and ROM. Fractures were classified by their location (greater tuberosity, metaphysis, diaphysis) and displacement.

All authors reviewed the postoperative radiographs for all patients who had a minimum 2-year followup $(n=29)$ and came to a consensus for each item analyzed. At the last clinical followup, radiographs were reviewed for presence of fracture union, humeral component loosening or subsidence, resorption or osteolysis, scapular notching, and postoperative fractures. Given that the majority of fractures were minimally displaced in the region of the greater tuberosity or metaphysis, the fractures were difficult to see on postoperative radiographs; therefore, the time to union was incalculable.

Humeral periprosthetic lucency was classified as 0 (none), 1 (< $1 \mathrm{~mm}$ wide, incomplete), 2 (1 $\mathrm{mm}$ wide, complete), 3 (1.5 mm wide, incomplete), 4 (1.5 $\mathrm{mm}$ wide, complete), or 5 (2 mm wide, complete).

\section{Statistical Analysis}

Categoric variables were assessed using the chi-square test (or Fisher's exact test) and unpaired t-tests were used for continuous variables when examining pre- and postoperative clinical outcomes. The Kaplan-Meier method was used to construct survival curves and compare overall survival between groups using the log-rank test. The odds ratio (OR) was calculated for each subgroup compared with the entire cohort $(n=230)$. Given the limited number of fractures and postoperative intraoperative fractures and complications among the 230 shoulders, our ability to perform multivariate analyses was restricted; therefore, each variable was assessed in a univariate fashion. All analyses were done using the $\mathrm{JMP}^{\circledR}$ statistical software package (Version 8; SAS Institute Inc, Cary, NC, USA), with a $\mathrm{p}$ value less than 0.05 considered statistically significant.

\section{Results}

Intraoperative Humerus Fractures

The cohort with intraoperative fractures included 27 women and nine men, with an average age of 72 years (range, 39-89 years). Average patient BMI was $31 \mathrm{~kg} / \mathrm{m}^{2}$, and the group included one laborer, two smokers, one patient with diabetes mellitus, and four patients with inflammatory arthritis. Of the 36 patients (36 shoulders), no patient had undergone a previous reverse arthroplasty, although 14 had prior anatomic shoulder arthroplasties and 22 had prior hemiarthroplasties. Two patients $(5 \%)$ had revision surgery for infections and 27 (75\%) had prior instability or subluxation, in addition to implant loosening. Eleven shoulders $(31 \%)$ had cemented humeral component primary arthroplasties, and nine shoulders (25\%) had a previous proximal humerus fracture, with two of the nine shoulders treated previously with open reduction internal fixation (ORIF). Previous stem lengths included 32 regular and four short stems. Only two patients had evidence of humeral component loosening in cementless stems on preoperative radiographs.

\section{Intraoperative Periprosthetic Humerus Fractures}

Intraoperative periprosthetic humerus fractures occurred in 36 of the 230 revision reverse shoulder arthroplasties (16\%) (Table 1). Twenty-nine $(81 \%)$ of the 36 intraoperative periprosthetic humerus fractures occurred during component removal and seven (19\%) occurred during preparation and impaction of the humeral component. Intraoperative fractures were treated with stabilization of the prosthetic stem in $28(78 \%)$ shoulders and adjunctive internal fixation in eight (22\%) shoulders. When no adjuvant fixation was used, the fractures were stabilized by a press-fit implant $(n=20)$ or a cemented stem $(n=8)$. The fractures treated with adjunctive fixation occurred in three patients with minimally displaced greater tuberosity fractures, one combined greater and lesser tuberosity minimally displaced fracture, two nondisplaced metaphyseal fractures, one displaced metaphyseal fracture, and one displaced metaphyseal fracture with extension in the diaphysis. Suture stabilization was used for the three greater tuberosity fractures and the combined greater and lesser tuberosity fracture. The two nondisplaced metaphyseal fractures were stabilized with cerclage wires alone. The two displaced metaphyseal fractures were stabilized using a strut allograft and cables. 
Table 1. Intraoperative fractures, characteristics, and treatment

\begin{tabular}{ll}
\hline Factor & $\begin{array}{l}\mathrm{n}=36 \text { patients } \\
(36 \text { shoulders })\end{array}$ \\
\hline Location & \\
Greater tuberosity & 30 \\
Metaphysis & 3 \\
Shaft & 3 \\
Periprosthetic fracture type & \\
Nondisplaced & 33 \\
Displaced & 3 \\
Timing of fracture & \\
Stem removal & 29 \\
Broaching of implant insertion & 7 \\
Implant length & \\
Short/micro & 10 \\
Standard & 25 \\
Long & 1 \\
Fracture stabilization & \\
Suture & \\
Luque wires & \\
Luque wires + bone graft & 22 \\
None & 28 \\
Use of cement in revision surgery & \\
Cemented & \\
Press-fit & \\
Difficult procedure modifier & \\
\hline & \\
&
\end{tabular}

All 36 fractures were classified as Type A according to the system described by Wright and Cofield [26]. There were 24 regular-length stems, 10 short stems, and one long stem used during the revision arthroplasties, with two patients receiving longer stems and eight receiving shorter stems than the humeral component implanted during the index surgery. Cement was used in $14(39 \%)$ of the revision arthroplasties complicated by intraoperative fractures, including nine of 14 with the cement-in-cement technique.

\section{Risk Factors for Fracture}

The risk of intraoperative periprosthetic fractures was increased by female sex (27 of 36 patients (75\%); OR, $2.41 ; 95 \% \mathrm{CI}, 1.11-5.68 ; \mathrm{p}=0.03)$, history of instability (27 of 36 patients (75\%); OR, 2.65; 95\% CI, 1.18-5.93; $\mathrm{p}=0.02$ ), and prior hemiarthroplasty ( 22 of 36 patients (61\%); OR, 2.34; 95\% CI, 1.13-4.84; $\mathrm{p}=0.03$ ) (Table 2). Previous proximal humerus fracture, previous ORIF, length of the revision stem, presence of preoperative humeral lucency, and use of cemented primary arthroplasties did not appear to be associated with risk of intraoperative humerus fracture (all $\mathrm{p}>0.6$ ).
Table 2. Risk factors for intraoperative humerus fractures

\begin{tabular}{lllr}
\hline Risk factor & OR & $95 \%$ CI & p value \\
\hline Difficult modifier & 3.73 & $1.71-8.12$ & $<0.01^{*}$ \\
History of instability & 2.65 & $1.18-5.93$ & $0.02^{*}$ \\
Female sex & 2.41 & $1.11-5.68$ & $0.03^{*}$ \\
Prior hemiarthroplasty & 2.34 & $1.13-4.84$ & $0.03^{*}$ \\
Inflammatory arthritis & 2.57 & $0.75-8.84$ & 0.13 \\
Cemented primary & 1.08 & $0.50-2.35$ & 0.84 \\
Age at surgery & 1.02 & $0.52-1.06$ & 0.21 \\
Prior proximal humerus fracture & 1.01 & $0.45-2.31$ & 0.97 \\
BMI & 0.94 & $0.89-1.01$ & 0.06 \\
Prior ORIF & 0.70 & $0.15-3.21$ & 0.65 \\
Preoperative humeral lucency & 0.70 & $0.15-3.21$ & 0.65 \\
Laborer & 0.53 & $0.03-2.88$ & 0.51 \\
Smoker & 0.43 & $0.10-0.94$ & 0.28 \\
Revision for infection & 0.40 & $0.09-1.76$ & 0.22 \\
Diabetes mellitus & 0.13 & $0.02-1.02$ & 0.052 \\
\hline
\end{tabular}

* Statistically significant at $\mathrm{p}<0.05 ; \mathrm{OR}=$ odds ratio; ORIF $=$ open reduction and internal fixation.

Postoperative Fractures, Revisions, Complications

Two postoperative periprosthetic fractures occurred in patients who had intraoperative fractures, including a nondisplaced greater tuberosity fracture in a patient with an intraoperative tuberosity fracture at 2 years postoperatively and a nondisplaced Type B (involving the stem) periprosthetic fracture in a patient with a previous intraoperative metaphyseal fracture at 1.1 years postoperatively. Both fractures were treated nonoperatively. Compared with the control group (no intraoperative fractures), there was no statistically significant difference $(p=0.13)$ in the incidence of postoperative fractures. In the control group, two postoperative periprosthetic fractures occurred; both were displaced Type $\mathrm{C}$ periprosthetic humerus fractures, one at 4 months and the other at 10 months postoperatively; both required ORIF with locking plates.

No patient with an intraoperative fracture underwent revision surgery for humeral component loosening. In the control group, one patient underwent revision surgery for humeral component loosening at 2 years postoperatively. The only other postoperative complication was a distal humerus fracture in a patient with an intraoperative nondisplaced fracture of the distal humerus.

Overall, there were three revision procedures performed in patients with a history of intraoperative fractures. Etiologies for revision surgery in patients with intraoperative periprosthetic fractures included glenoid loosening $(n=2)$ and instability $(\mathrm{n}=1)$. The 2 - and 5-year survivorship estimates for patients with an intraoperative fracture were $94 \%( \pm 6)$ and $85 \%( \pm 11)$, respectively, compared with 


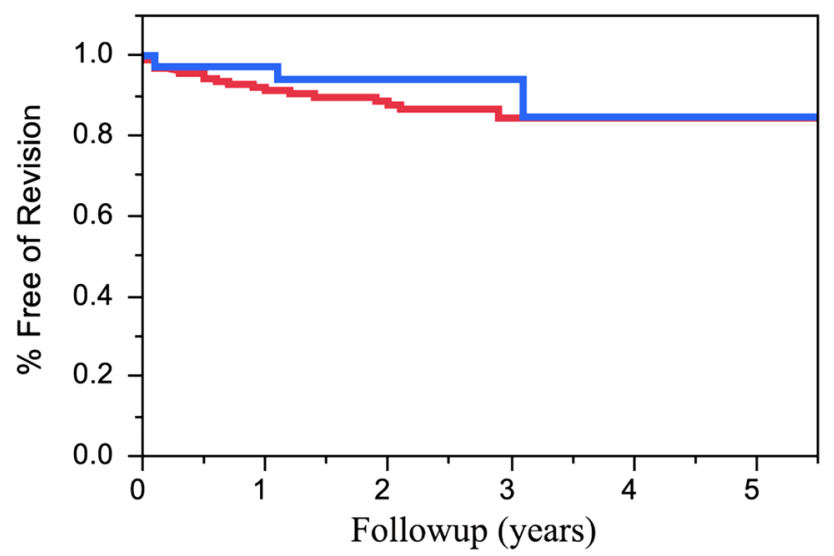

Fig. 1 At an average followup of 3.1 years, three of 36 patients (8\%) underwent revision surgery after sustaining an intraoperative fractures. The 2- and 5-year survival rates for patients with an intraoperative fracture (blue) were $94 \%( \pm 6 \%)$ and $85 \%( \pm 11 \%)$, respectively, compared with $89 \%( \pm 4 \%)$ and $84 \%( \pm 6)$ for patients without an intraoperative fracture (red; $\mathrm{p}=0.45$ ).

$89 \%( \pm 4)$ and $84 \%( \pm 6)$, respectively, for patients without an intraoperative fracture $(\mathrm{p}=0.45)$ (Fig. 1). The need for fracture fixation or displacement of the fractures did not influence the likelihood of subsequent revision surgery $(\mathrm{p}=0.34)$. Three $(8 \%)$ patients with intraoperative fractures had humeral lucency at last followup. There was no difference in humeral lucency compared with the control group (without intraoperative fractures), with two patients having Grade 1 or 2 lucency in the fracture group $(\mathrm{n}=29)$ compared with five patients having Grade 1 or 2 humeral lucency and one having Grade 3 lucency in the control group $(p=0.45)$. No patients with an intraoperative fracture had evidence of humeral component subsidence.

\section{Clinical Outcomes}

Of the 29 patients with more than 2 years followup, 27 experienced pain relief after revision arthroplasty, with $90 \%$ reporting moderate or severe pain preoperatively compared with $7 \%$ postoperatively $(\mathrm{p}<0.001)$. Shoulder abduction also improved from $47^{\circ}$ preoperatively to $115^{\circ}$ postoperatively $(\mathrm{p}<0.01)$. Patients experienced reasonable postoperative American Shoulder and Elbow Surgeons and Simple Shoulder Test scores. There was no difference between the patient groups regarding postoperative pain levels, shoulder abduction, American Shoulder and Elbow Surgeons, and Simple Shoulder Test scores ( $p>$ 0.35)(Table 3). The postoperative rehabilitation protocol was not modified in any of the patients who experienced an intraoperative fracture.
Table 3. Clinical outcomes

\begin{tabular}{lcc}
\hline Outcome measure & Rating & p value \\
\hline $\begin{array}{l}\text { Pain (moderate or severe), n (\%) } \\
\quad\end{array}$ & $2(7)$ & 0.76 \\
Intraoperative fracture & $21(9)$ & \\
$\quad$ No intraoperative fracture & & 0.71 \\
Shoulder abduction, mean (range) & & \\
$\quad$ Intraoperative fracture & $115(30-180)$ & \\
No intraoperative fracture & $119(10-160)$ & 0.88 \\
ASES, mean score (range) & & \\
Intraoperative fracture & $65.0(30-98)$ & \\
$\quad$ No intraoperative fracture & $64.7(18-98)$ & 0.35 \\
SST, positive response & & \\
Intraoperative fracture & $6.2(3-11)$ & \\
No intraoperative fracture & $5.9(0-11)$ & \\
\hline
\end{tabular}

ASES $=$ American Shoulder and Elbow Surgeons; SST $=$ Simple Shoulder Test.

\section{Discussion}

Studies examining intraoperative complications, such as periprosthetic fractures $[2,11,12,16,20,22-24]$ during revision reverse shoulder arthroplasty have been done. There is a relatively high frequency of early complications associated with the procedure, partly attributable to the technical demands of the surgery and its relatively recent approval for use (2004) in the United States [2, 11, 16, 20, $22,23]$. Therefore, the purposes of our study were to assess the frequency, risk factors, and early outcomes associated with intraoperative periprosthetic humerus fractures in patients undergoing revision reverse shoulder arthroplasty.

There were several limitations to the study. Although our study had a relatively large cohort of patients who underwent reverse shoulder arthroplasty, the number of patients was much smaller than in similar studies of periprosthetic fractures in patients having anatomic shoulder arthroplasty [1, 15, 19]. Furthermore, given the small number of intraoperative fractures that involved the metaphysis or diaphysis, it is difficult to draw conclusions regarding the optimal fixation method across all possible fracture types. However, given the high number of revision surgeries performed at our institution, despite the small cohort size, our study provides a glimpse into how to evaluate and treat these intraoperative fractures when they do occur. Although we were able to analyze early outcomes, the low number of patients with relatively recent fractures prevented us from commenting on long-term effects of intraoperative humerus fractures.

Another limitation was that the series involved patients from one institution, limiting our ability to generalize our findings to other cohorts. This also introduced a referral bias; however, it also eliminated other confounding 
variables seen when patient groups from multiple institutions are involved in studies. Another limitation of our study was that seven patients who experienced intraoperative fractures were lost to followup. Therefore, our conclusions regarding the outcomes of postoperative fractures are limited to the 29 patients who had more than 2 years of clinical followup. Finally, an unknown number of patients did not consent for study in our institution's registry; however, traditionally this number is thought to be less than $1 \%$ of all patients treated at our institution.

\section{Frequency and Characteristics of Fractures}

Our series of 230 consecutive reverse shoulder arthroplasties performed in the revision setting showed that intraoperative humerus fractures occur in approximately $16 \%$ of procedures. In our series of revision reverse TSAs, the frequency of intraoperative fractures was higher than the reported frequency during revision arthroplasty with an anatomic component [1].

The majority of the intraoperative fractures occurred during implant removal using standard implant removal techniques such as osteotomes or a pencil-tip burr to separate the proximal implant-bone interface. No osteotomies were required. The majority of the fractures were isolated to the greater tuberosity. Only eight fractures were treated with adjunctive internal fixation, with cerclage wires used in two of the nondisplaced metaphyseal fractures, cerclage wires and bone graft used in the displaced metaphyseal fracture, and strut allograft with cerclage wire fixation used in a displaced fracture that extended into the diaphysis. In comparison, Athwal et al. [1] examined 45 intraoperative periprosthetic fractures in primary and revision anatomic should arthroplasties. Nineteen of the fractures involved the greater tuberosity, 16 involved the humeral shaft, three involved the greater tuberosity with extension into the humeral shaft, six were localized to the metaphysis, and one was a greater and lesser tuberosity fracture.

\section{Risks of Fractures}

Female sex, prior instability, and patients with a prior hemiarthroplasty were at increased risk for intraoperative periprosthetic humerus fractures. Several studies have identified factors associated with increased risk of periprosthetic fractures, including osteopenia, female sex, revision surgery, rheumatoid arthritis, overreaming or using an oversized broach in the humeral component preparation, humeral deformity, and soft tissue contracture $[1,3-7,10,15,26]$. Furthermore, female patients were 2.5 times more likely to experience an intraoperative periprosthetic fracture than male patients, potentially secondary to the porosity of their bones. Likewise patients with inflammatory arthritis and those with primary hemiarthroplasties were more than two times more likely to experience an intraoperative periprosthetic fracture.

\section{Complications and Outcomes of Intraoperative Fractures}

The only postoperative periprosthetic fractures that occurred in patients with an intraoperative fracture included a nondisplaced greater tuberosity fracture in a patient with an intraoperative tuberosity fracture and a nondisplaced Type B periprosthetic fracture in a patient with a previous intraoperative metaphyseal fracture. The two other fractures occurred in patients without an intraoperative fracture. No patient underwent revision surgery for humeral component loosening. There was no difference in early survival rates between patients with and without intraoperative fractures. The need for intraoperative stabilization did not influence postoperative outcomes, similar to other studies of anatomic shoulder arthroplasty $[1,7,21,26]$. As all of the fractures in our series were classified as Type A (localized to the greater tuberosity) [26], without any extension into the humeral diaphysis, long-stemmed implants were not needed; however, as suggested by others $[1,7,21,26]$, we recommend a systemic approach to treating these fractures according to the location, displacement, and bone quality.

Patients whose surgeries were complicated by intraoperative fractures experienced excellent pain relief and improved shoulder ROM, with reasonable postoperative American Shoulder and Elbow Surgeons and Constant and Murley shoulder scores. Our series showed the relatively high incidence of periprosthetic humerus fractures during revision shoulder arthroplasty using a reverse prosthesis. However, this intraoperative complication does not appear to negatively affect early postoperative outcomes.

Although intraoperative periprosthetic fractures have been thoroughly examined in anatomic shoulder arthroplasties, there is a paucity of information regarding revision reverse shoulder arthroplasties. An important finding involves eliciting the risks associated with these fractures, including female sex, presence of inflammatory arthritis, and hemiarthroplasty implant removal. Furthermore, intraoperative periprosthetic fractures do not always need adjunctive fixation and do not appear to affect postoperative outcomes for patients. We recommend a methodic approach to fracture evaluation and treatment based on the perceived humerus implant stability. If needed, a long-stem implant or adjunct fixation (plate, screws, wires) should be used if the humerus implant stability is in question. However, in our series, minimally or nondisplaced fractures in the region of the 
tuberosities often did not require fixation and did not seem to have a substantial effect on postoperative outcomes. When these fractures are approached in a systematic way to achieve anatomic reduction and stable fixation, they do not have a substantial effect on overall final outcome.

This study provides a foundation for future investigations of risk reduction through modification of surgical techniques and/or patient risk factors. Furthermore, when removing implants, a systemic approach should be used, starting with an implant-specific removal device and other techniques to separate the proximal implant-bone interface, to help mitigate the risks of intraoperative periprosthetic fractures.

Acknowledgment Scott P. Steinmann MD (Department of Orthopedic Surgery, Mayo Clinic, Rochester, MN, USA) was among the five fellowship-trained surgeons who performed the surgeries reported in this study.

\section{References}

1. Athwal GS, Sperling JW, Rispoli DM, Cofield RH. Periprosthetic humeral fractures during shoulder arthroplasty. J Bone Joint Surg Am. 2009;91:594-603.

2. Boileau P, Watkinson DJ, Hatzidakis AM, Balg F. Grammont reverse prosthesis: design, rationale, and biomechanics. $J$ Shoulder Elbow Surg. 2005;14(1 suppl S):147S-161S.

3. Bonutti PM, Hawkins RJ. Fracture of the humeral shaft associated with total replacement arthroplasty of the shoulder: a case report. J Bone Joint Surg Am. 1992;74:617-618.

4. Boyd AD Jr, Thornhill TS, Barnes CL. Fractures adjacent to humeral prostheses. J Bone Jont Surg Am. 1992;74:1498-1504.

5. Brenner BC, Ferlic DC, Clayton ML, Dennis DA. Survivorship of unconstrained total shoulder arthroplasty. J Bone Joint Surg Am. 1989;71:1289-1296.

6. Cameron B, Iannotti JP. Periprosthetic fractures of the humerus and scapula: management and prevention. Orthop Clin North Am. 1999;30:305-318.

7. Campbell JT, Moore RS, Iannotti JP, Norris TR, Williams GR. Periprosthetic humeral fractures: mechanisms of fracture and treatment options. J Shoulder Elbow Surg. 1998;7:406-413.

8. Chin PY, Sperling JW, Cofield RH, Schleck C. Complications of total shoulder arthroplasty: are they fewer or different? J Shoulder Elbow Surg. 2006;15:19-22.

9. Cofield RH. Total shoulder arthroplasty with the Neer prosthesis. J Bone Joint Surg Am. 1984;66:899-906.

10. Cuomo F, Checroun A. Avoiding pitfalls and complications in total shoulder arthroplasty. Orthop Clin North Am. 1998;29:507-518.
11. Frankle MA, Teramoto A, Luo ZP, Levy JC, Pupello D. Glenoid morphology in reverse shoulder arthroplasty: classification and surgical implications. J Shoulder Elbow Surg. 2009;18:874-885.

12. Groh GI, Heckman MM, Wirth MA, Curtis RJ, Rockwood CA Jr. Treatment of fractures adjacent to humeral prostheses. J Shoulder Elbow Surg. 2008;17:85-89.

13. Hattrup SJ, Sanchez-Sotelo J, Sperling JW, Cofield RH. Reverse shoulder replacement for patients with inflammatory arthritis. J Hand Surg Am. 2012;37:1888-1894.

14. Klein SM, Dunning P, Mulieri P, Pupello D, Downes K, Frankle MA. Effects of acquired glenoid bone defects on surgical technique and clinical outcomes in reverse shoulder arthroplasty. J Bone Joint Surg Am. 2010;92:1144-1154.

15. Kumar S, Sperling JW, Haidukewych GH, Cofield RH. Periprosthetic humeral fractures after shoulder arthroplasty. J Bone Joint Surg Am. 2004;86:680-689.

16. Levy J, Frankle M, Mighell M, Pupello D. The use of the reverse shoulder prosthesis for the treatment of failed hemiarthroplasty for proximal humeral fracture. J Bone Joint Surg Am. 2007;89: 292-300.

17. Matsen FA 3rd, Ziegler DW, DeBartolo SE. Patient self-assessment of health status and function in glenohumeral degenerative joint disease. J Shoulder Elbow Surg. 1995;4:345-351.

18. Neer CS 2nd, Watson KC, Stanton FJ. Recent experience in total shoulder replacement. J Bone Joint Surg Am. 1982;64:319-337.

19. Singh JA, Sperling J, Schleck C, Harmsen W, Cofield R. Periprosthetic fractures associated with primary total shoulder arthroplasty and primary humeral head replacement: a thirtythree-year study. J Bone Joint Surg Am. 2012;94:1777-1785.

20. Sirveaux F, Favard L, Oudet D, Huquet D, Walch G, Mole D. Grammont inverted total shoulder arthroplasty in the treatment of glenohumeral osteoarthritis with massive rupture of the cuff: results of a multicentre study of 80 shoulders. J Bone Joint Surg Br. 2004;86:388-395.

21. Steinmann SP, Cheung EV. Treatment of periprosthetic humerus fractures associated with shoulder arthroplasty. J Am Acad Orthop Surg. 2008;16:199-207.

22. Werner CM, Steinmann PA, Gilbart M, Gerber C. Treatment of painful pseudoparesis due to irreparable rotator cuff dysfunction with the Delta III reverse-ball-and-socket total shoulder prosthesis. J Bone Joint Surg Am. 2005;87:1476-1486.

23. Wierks C, Skolasky RL, Ji JH, McFarland EG. Reverse total shoulder replacement: intraoperative and early postoperative complications. Clin Orthop Relat Res. 2009;467:225-234.

24. Wirth MA, Rockwood CA Jr. Complications of shoulder arthroplasty. Clin Orthop Relat Res. 1994;307:47-69.

25. Worland RL, Kim DY, Arredondo J. Periprosthetic humeral fractures: management and classification. J Shoulder Elbow Surg. 1999;8:590-594.

26. Wright TW, Cofield RH. Humeral fractures after shoulder arthroplasty. J Bone Joint Surg Am. 1995;77:1340-1346. 